\title{
Functional neuroimaging in Tourette syndrome: recent perspectives
}

\author{
This article was published in the following Dove Press journal: \\ Neuroscience and Neuroeconomics \\ 26 April 2017 \\ Number of times this article has been viewed
}

\section{Nanette Mol Debes \\ Marie Préel \\ Liselotte Skov}

Pediatric Department, Tourette Clinic, Herlev University Hospital, Herlev, Denmark
Correspondence: Nanette Mol Debes Pediatric Department, Tourette Clinic, Herlev University Hospital, Nystedvej 9, 2500 Valby, Denmark

Tel +4521656580

Email nanettemol@hotmail.com

\begin{abstract}
The most recent functional neuroimaging studies on Tourette syndrome (TS) are reviewed in this paper. Although it can be difficult to compare functional neuroimaging studies due to differences in methods, differences in age of the included subjects, and differences in the extent to which the presence of comorbidity, medical treatment, and severity of tics are considered in the various studies; most studies show that the cortico-striato-thalamo-cortical circuit seems to be involved in the generation of tics. Changes in this circuit seem to be correlated with tic severity. Correlations have been found between the presence of tics and hypermetabolism in various brain regions. Abnormalities of GABAergic, serotonergic, and dopaminergic neurotransmission in patients with TS have been suggested. During tic suppression, increased activity in the inferior frontal gyrus is seen. The premotor cortex might be involved in inhibition of motor control in subjects with TS. The right anterior insula is suggested to be a part of the urge-tic network. Several studies have shown altered motor network activations and sensorimotor gating deficits in subjects with TS. In future studies, inclusion of more well-defined subjects and further examination of premonitory urge and tic suppression is needed in order to increase the knowledge about the pathophysiology and treatment possibilities of TS.
\end{abstract}

Keywords: functional neuroimaging, Tourette syndrome

\section{Introduction}

Tourette syndrome (TS) is a hereditary, chronic, neurobiological disease characterized by the presence of motor and vocal tics. Tics are sudden, rapid, recurrent, nonrhythmic movements (motor tics) or sounds (vocal tics). ${ }^{1}$ They usually start before the age of 12 years and wax and wane (ie, increase and decrease in severity over time). Tics are suppressible and are usually preceded by premonitory urges, which is an unpleasant sensation in the body. Patients often report a sense of relief after tics. ${ }^{2}$ Premonitory urge can be measured by using the Premonitory Urges for Tics Scale (PUTS). ${ }^{3}$ PUTS has been found to have a significant correlation with tic severity and quality of life. ${ }^{4}$ Furthermore, premonitory urge plays a role in the more recent behavioral therapies used in the treatment of TS. ${ }^{5}$

TS is often accompanied by other symptoms. The two best-known comorbidities are obsessive-compulsive disorder (OCD) and attention deficit hyperactivity disorder (ADHD), but other symptoms such as depression, outbursts of rage, and sleeping disturbances are often seen. ${ }^{6}$

Recent reviews $\mathrm{s}^{2,7,8}$ have estimated the prevalence of TS to be around $0.8 \%$ (range: $0.3-5.7)$, with a predominance in boys $(4.3: 1){ }^{9}$ 
The etiology of TS is not yet fully understood, and genetic, environmental, and neuroanatomical factors may play a role. During recent years, focus on neuroimaging studies has increased. The cortico-striato-thalamo-cortical pathways seem to be involved in the pathophysiology of TS, but the results of many neuroimaging studies are conflicting. Among others, differences in methods could be the reason for contradictory results.

In functional neuroimaging, imaging technology is used in order to increase the understanding of the relationship between activity in brain areas and specific functions. The interaction between brain regions in neural processing, the time-course of neural events, and the location of neural events are some of the aspects that can be measured with functional neuroimaging. Since several brain regions and pathways seem to be involved in the pathophysiology of TS, this is a promising method in the field of TS research.

In this paper, we review the functional neuroimaging studies on TS from the last 10 years, ie, from 2006 to 2016. Since the number of neuroimaging studies has expanded during the last years, we decided to only include the studies from the last 10 years in order to review the most updated knowledge on this topic. We have chosen to include the following functional neuroimaging methods: $:^{10}$ functional magnetic resonance imaging (fMRI) studies, magnetoencephalography (MEG), functional connectivity (FC), transcranial magnetic stimulation (TMS), magnetic resonance spectroscopy (MRS), near-infrared spectroscopy (NIRS), positron emission tomography (PET), single-photon emission computed tomography (SPECT), and electroencephalography (EEG).

We have used PubMed using the search terms "Tourette syndrome and functional neuroimaging" and "Tourette syndrome and [the specific above mentioned functional neuroimaging methods]", and relevant articles via references from the found articles published in the period 2006-2016.

The most important neuroimaging findings are summarized in Table 1 and are described more detailed in the sections below. Figure 1 shows a simplified diagram of the neurocircuitry involved in the pathophysiology of TS.

Table I Summary of the most important findings from functional neuroimaging studies on TS from the last 10 years, ie, from 2006 to 2016.

\begin{tabular}{|c|c|c|}
\hline $\begin{array}{l}\text { Neural events } \\
\text { and functions }\end{array}$ & $\begin{array}{l}\text { Functional } \\
\text { neuroimaging } \\
\text { method }\end{array}$ & Involved structures in patients with TS \\
\hline \multirow[t]{3}{*}{ Premonitory urges } & fMRI & I. Involvement of insula and cingulate cortex ${ }^{15}$ \\
\hline & & $\begin{array}{l}\text { 2. Gray matter thinning within sensory areas of the frontosubcortical circuitry, }{ }^{15} \text { within the left insula and } \\
\text { right sensorimotor cortex }{ }^{17}\end{array}$ \\
\hline & PET & Prominent activation in cerebellum and insula ${ }^{60}$ \\
\hline \multirow[t]{7}{*}{ Tic generation } & fMRI & $\begin{array}{l}\text { I. Two seconds before tic: activation of supplementary motor area, ventral primary motor cortex, primary } \\
\text { sensorimotor cortex, parietal operculum, }{ }^{12} \text { and anterior cingulate cortex }{ }^{13}\end{array}$ \\
\hline & & $\begin{array}{l}\text { 2. One second before a tic: activation of anterior cingulate cortex, putamen, insula, amygdala, cerebellum, } \\
\text { and the extrastriatal visual cortex }\end{array}$ \\
\hline & & $\begin{array}{l}\text { 3. During tic onset: activation in the thalamus, central operculum, primary motor and somatosensory } \\
\text { cortices }^{\prime 2} \text {, as well as in sensorimotor areas and cerebellum }{ }^{13}\end{array}$ \\
\hline & & 4. Involvement of sensorimotor cortex" \\
\hline & MEG & Involvement of sensorimotor cortex" \\
\hline & EEG & Spreading of neural oscillation from the left prefrontal and centroparietal regions to the left frontal regions ${ }^{70}$ \\
\hline & PET & Prominent activation in cerebellum and insula ${ }^{60}$ \\
\hline $\begin{array}{l}\text { Voluntary } \\
\text { expression of tics }\end{array}$ & EEG & Increased oscillations in the prefrontal region ${ }^{74}$ \\
\hline \multirow[t]{2}{*}{ Tic suppression } & fMRI & Increased activity in left inferior frontal gyrus ${ }^{19}$ \\
\hline & EEG & Overactivation of cortical areas ${ }^{71}$ \\
\hline Inhibitory motor & fMRI & I. Decreased activity in left primary motor cortex and secondary motor areas during go trials $\mathrm{s}^{22}$ \\
\hline \multirow{6}{*}{ control } & & 2. Reduced activation in the dorsal premotor cortex for the StopSuccess ${ }^{21}$ \\
\hline & & 3. Activation of prefrontal cortex during regulation of motor output ${ }^{24}$ \\
\hline & & 4. Sensorimotor gating deficits during prepulse inhibition 28,29 \\
\hline & & 5. Involvement of frontostriatal circuits in self-regulatory task $\mathrm{k}^{25,26}$ \\
\hline & & $\begin{array}{l}\text { 6. Increased activation in the direct pathway through the basal ganglia and increased compensatory activation } \\
\text { in the prefrontal cortex and subthalamic nucleus during the performance of a cognitive control task }\end{array}$ \\
\hline & MEG & I. Sensorimotor cortex ${ }^{28}$ \\
\hline
\end{tabular}


Table I (Continued)

\begin{tabular}{|c|c|c|}
\hline & $\begin{array}{l}\text { Functional } \\
\text { neuroimaging } \\
\text { method }\end{array}$ & Involved structures in patients with TS \\
\hline \multirow{7}{*}{$\begin{array}{l}\text { Voluntary motor } \\
\text { execution }\end{array}$} & fMRI & I. Decreased regional volumes of cerebellar hemispheres during finger tapping ${ }^{33}$ \\
\hline & & 2. Altered motor network activations during simple motor performance ${ }^{32}$ \\
\hline & & 3. Different neurofunctional organization of motor control $^{34}$ \\
\hline & & 4. No differences in activation patterns during finger tapping task ${ }^{30}$ \\
\hline & MEG & I. Involvement of sensorimotor cortex ${ }^{39}$ \\
\hline & & 2. Increased interaction between supplementary motor area and primary motor cortex ${ }^{40}$ \\
\hline & & 3. Altered motor cortical reactivity ${ }^{41}$ \\
\hline \multirow[t]{2}{*}{$\begin{array}{l}\text { Perception and } \\
\text { listening }\end{array}$} & fMRI & $\begin{array}{l}\text { I. Deficient frontal inhibition in the interaction of the cortico-striato-thalamo-cortical network within } \\
\text { structures of the limbic system }{ }^{36}\end{array}$ \\
\hline & & 2. Different callosal functioning during listening ${ }^{37}$ \\
\hline \multirow[t]{20}{*}{ Resting state fMRI } & fMRI & Abnormal spontaneous neuronal activity and perturbed connectivity of cortico-subcortical networks ${ }^{38}$ \\
\hline & $\begin{array}{l}\text { Functional } \\
\text { connectivity }\end{array}$ & $\begin{array}{l}\text { I. Abnormal functional connectivity in the bilateral prefronto-striatum-midbrain networks and in the } \\
\text { bilateral sensorimotor and temporal cortices }{ }^{42}\end{array}$ \\
\hline & & 2. Altered functional connectivity of amygdala ${ }^{43}$ \\
\hline & & 3. Abnormal signaling primarily in the frontoparietal network ${ }^{44}$ \\
\hline & & 4. Stronger functional integration and a global functional disorganization of corticobasal ganglia networks ${ }^{46}$ \\
\hline & TMS & I. Abnormal functional interhemispheric connectivity ${ }^{51}$ \\
\hline & & 2. Increased resting motor threshold ${ }^{52}$ \\
\hline & & 3. Increased variability of motor evoked potential responses ${ }^{52}$ \\
\hline & MRS & I. Reduced primary sensorimotor cortex GABA concentrations $s^{53}$ \\
\hline & & 2. No differences in GABA values in the right sensorimotor cortex" \\
\hline & & 3. Increased GABA concentrations within the supplementary motor area ${ }^{55}$ \\
\hline & NIRS & Reduced prefrontal hemodynamic response $\mathrm{e}^{56}$ \\
\hline & PET & I. Hypermetabolism in various brain regions $s^{57-59}$ \\
\hline & & 2. Decreased binding of GABA receptors ${ }^{54}$ \\
\hline & & 3. Abnormal dopaminergic neurotransmission ${ }^{61-64}$ \\
\hline & & 4. Normal dopamine receptor availability and affinity ${ }^{65,66}$ \\
\hline & & 5. Abnormal serotonergic neurotransmission $n^{64,67-69}$ \\
\hline & SPECT & I. DAT uptake ratios were significantly negatively correlated with disease duration ${ }^{70}$ \\
\hline & & 2. Functional abnormality of the dopamine system in patients with TS in its early stage $\mathrm{e}^{71}$ \\
\hline & & 3. Normal DAT activity ${ }^{71,72}$ \\
\hline
\end{tabular}

Abbreviations: TS, Tourette syndrome, fMRI, functional magnetic resonance imaging; MEG, magnetoencephalography; MRS, magnetic resonance spectroscopy; NIRS, near infrared spectroscopy; PET, positron emission tomography; EEG, electroencephalography; GABA, gamma-amino-butyric acid; DAT, dopamine transporter; SPECT, singlephoton emission computed tomography.

\section{fMRI}

fMRI uses task-based changes in blood flow measured by blood-oxygen-level-dependent contrast in order to examine neural activity in the brain.

\section{Tics, premonitory urges, and tic suppression \\ Tics}

Several studies have examined the tic-related activity in the brain. ${ }^{11,12}$ The temporal pattern of tic generation was found to follow the cortico-striato-thalamo-cortical circuit. ${ }^{12}$ Several studies found activation in the supplementary motor area (SMA), ventral primary motor cortex, primary sensorimotor cortex, the parietal operculum, ${ }^{12,13}$ and anterior cingulate cortex ${ }^{13} 2 \mathrm{~s}$ before tic onset. One second before a tic, the anterior cingulate cortex, putamen, insula, amygdala, cerebellum, and the extrastriatal-visual cortex showed activation. ${ }^{12}$ During tic onset, activation in the thalamus, central operculum, primary motor and somatosensory cortices was found, ${ }^{12}$ as well as in sensorimotor areas and cerebellum. ${ }^{13}$ The involvement of SMA and sensorimotor processing disturbances in tic generation was confirmed by several studies. ${ }^{11,14}$ Hampson et $\mathrm{l}^{14}$ found that SMA showed a significantly broader profile of cross-correlation to motor cortex during tics than during intentional movements. Tinaz et $\mathrm{al}^{11}$ found that the anterior insula showed increased FC with the sensorimotor cortex in patients with TS.

\section{Premonitory urges}

Several studies have shown that the insula, cingulate cortex, and gray matter thinning within sensory areas of the frontosubcortical circuitry are involved in the pathophysiology of generation of premonitory urges (reviewed by Rajagopal et $\left.\mathrm{al}^{15}\right)$. 


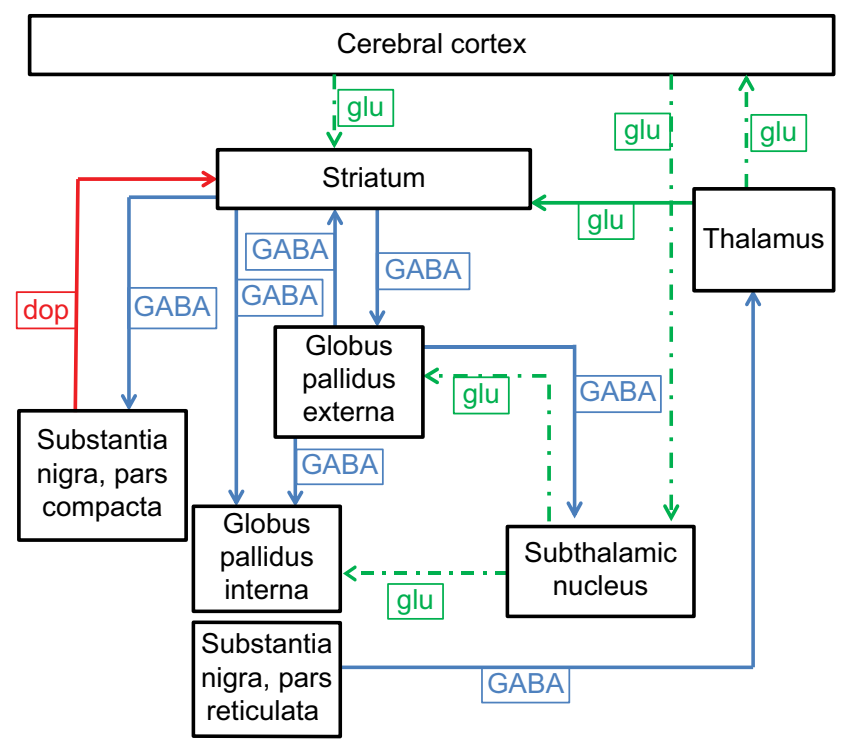

Figure I Simplified diagram of the cortico-striato-thalamo-cortical circuit. Notes: Inhibitory connections are marked by continuous lines; excitatory connections are marked by dotted lines. Green indicates connections that use glu as neurotransmitter; blue indicates connections that use GABA as neurotransmitter; red indicates connections that use dop as neurotransmitter. In the presence of dopamine, dopamine receptors in the basal ganglia stimulate the GABAergic neurons. Reprinted from Movement Disorders in Childhood, 2nd ed, Singer HS, Mink JW, Gilbert DL, Jankovic J, Copyright 2016, with permission from Elsevier. ${ }^{92}$ Abbreviations: dop, dopamine; glu, glutamate.

One study found higher connectivity between the right dorsal anterior insula and the frontostriatal nodes of the urge-tic network in patients with TS compared with healthy controls. ${ }^{16}$ The FC between the right dorsal anterior insula and SMA area correlated positively with urge severity. ${ }^{16}$

A more recent study has shown that premonitory urges are associated with a significantly reduced gray matter thickness within the left insula and right sensorimotor cortex. ${ }^{17}$

\section{Tic suppression}

Several studies examined the neural correlates of tic suppression. Kawohl et al ${ }^{18}$ did not find different activation of the anterior cingulate cortex under the conditions suppression of tics, tics, or imitation of tics. Ganos et al ${ }^{19}$ found increased activity in the left inferior frontal gyrus during voluntary tic inhibition compared with free ticcing. Mazzone et $\mathrm{al}^{20}$ studied neural correlates of inhibition of the semivoluntary motor behavior eye blinking. TS patients activated the frontal cortex and striatum more strongly during eye blinking inhibition than healthy controls. The authors suggested that the activation of frontostriatal circuits might help to maintain control over semivoluntary behaviors, whether there are tics or eye blinks.

In summary, fMRI studies have shown that the corticostriato-thalamo-cortical circuit and sensorimotor cortex seem to be involved in the generation of tics and that the insula and sensorimotor cortex seem to be a part of the urge-tic network. During tic suppression, increased activity in the inferior frontal gyrus was seen.

\section{Functional correlates of inhibitory motor control}

Several fMRI studies have used tasks of inhibitory motor control such as the go/no-go task in patients with TS. $.^{21,22} \mathrm{Go} /$ no-go test is a test of behavioral control in which an appropriate reaction must be performed and an inappropriate reaction must be inhibited. Results are contradictory. Thomalla et $\mathrm{al}^{22}$ found decreased activity in left primary motor cortex and secondary motor areas during go trials. Another study showed a reduced activation in the dorsal premotor cortex for the StopSuccess trial. ${ }^{21}$ The conflicting results might be caused by a difference in study design: in Ganos et al's study, ${ }^{21}$ the procedure was adapted to ensure a fifty-fifty successful performance in the no-go trials in both patients and controls, whereas in Thomalla et al's study, ${ }^{22}$ there were behavioral differences between patients and controls. The patients with TS were slower and and made more mistakes in no-go trials than the healthy controls. ${ }^{23}$

To examine behavioral regulation of motor output in children and adolescents with TS, Jackson et $\mathrm{a}^{24}$ used a manual task-switching paradigm and found a greater activation of prefrontal cortex in patients with TS compared with healthy controls when carrying out the task. Several studies have examined the neural correlates of self-regulatory tasks and found involvement of frontostriatal circuits. ${ }^{25,26}$ Increased activation in the direct pathway through the basal ganglia and increased compensatory activation in the prefrontal cortex and subthalamic nucleus during the performance of a cognitive control task was found in another study. ${ }^{27}$

Two studies have investigated the neural correlates of prepulse inhibition (PPI). ${ }^{28,29} \mathrm{PPI}$ is a neurological phenomenon in which a weaker prestimulus (prepulse) inhibits the reaction of an organism to a subsequent strong startling stimulus (pulse). A couple of studies indicate sensorimotor gating deficits in patients with TS. ${ }^{28,29}$ Buse et al ${ }^{29}$ showed increased activity in five regions of the brain during PPI (middle frontal gyrus, the postcentral gyrus, the superior parietal cortex/ precuneus, the anterior cingulated gyrus, and the caudate body), and they suggested that this deficient sensorimotor gating in patients with TS might be associated with reduced recruitment of brain regions responsible for the higher-order integration of somatosensory stimuli.

In summary, the premotor cortex might be involved in inhibition of motor control in patients with TS, but the results are contradictory. There seems to be evidence for sensorimotor gating deficits in TS. Furthermore, activation 
of the prefrontal cortex might be increased during regulation of motor output in TS.

\section{Functional correlates of voluntary motor execution}

Several studies have examined the neural correlates for a finger-tapping task in patients with TS. ${ }^{30-33}$ Tobe et al ${ }^{33}$ found decreased regional volumes of the cerebellar hemispheres during a finger-tapping test in patients with TS. The authors hypothesize that the cerebellum might be involved in the pathogenesis of TS. Roessner et $\mathrm{al}^{32}$ found a higher recruitment of the inferior parietal lobe during right (preferred) and left index finger tapping in TS compared with healthy controls. Compared with controls, less recruitment of the caudate and middle frontal gyrus was seen during preferred right finger tapping, and during left (nonpreferred) index finger tapping, less activity in contralateral right SM1 (primary sensorimotor cortex) together with greater activation of several other brain regions was seen. These results indicate that children with TS might show altered motor network activations during simple motor performance. These alterations were more pronounced when finger movements were executed with the nonpreferred left hand. In a preceding study, based on movements of the right index finger, the same authors found reduced premotor and prefrontal activations and increased activations of the right medial frontal gyrus and the left caudate in patients with TS compared with healthy controls. ${ }^{31}$ The hemispheric preponderance of this task might be the reason for this discrepancy. ${ }^{32}$

In contrast, one study did not find any differences in activation patterns during a finger-tapping task between children with TS and healthy controls. ${ }^{30}$

One study examined whether there was a difference in activation pattern of imaginary and actual voluntary finger oppositions in patients with TS and healthy controls. ${ }^{34}$ In both actual and imaginary motor tasks, increased activation was seen in patients with TS. During actual motor tasks, increased activation was seen in the premotor and prefrontal areas, and during imaginary motor tasks, hyperactivation was seen in the rostral prefrontal and temporoparietal regions of the right hemisphere. These findings suggest a different neurofunctional organization of motor control between patients with TS and healthy controls that is independent from the actual execution of motor acts.

Margolis et $\mathrm{al}^{35}$ measured performance on a verbalmanual interference task in which performance on a manual motor task at baseline was compared with performance of the same task concurrently with a hemispherically lateralized cognitive task. The left-hand performance was better in the healthy controls compared with the patients with TS, and this was accompanied by larger prefrontal cortex volumes.

In summary, most studies showed altered motor network activations during voluntary motor execution in patients with TS.

\section{Functional correlates of perception and listening}

Neuner et $\mathrm{al}^{36}$ investigated the perception of six emotional facial expressions in adults with TS. Compared with healthy controls, significantly higher amygdala activation was found in patients with TS. The authors suggest that this could be caused by deficient frontal inhibition due to structural changes or a desynchronization in the interaction of the cortico-striato-thalamo-cortical network within structures of the limbic system.

Plessen et $\mathrm{al}^{37}$ found indications for a different callosal functioning in the TS group compared with healthy controls while performing verbal dichotic listening when focusing attention on a left ear stimulus. Focus on right ear stimulus was thought to involve the same prefrontal attention and executive functions that are involved in the suppression of tics, whereas focus on left ear stimulus additionally was thought to involve a callosal transfer of information.

In summary, deficient frontal inhibition and changes in callosal functioning might be involved in the perception of some senses in patients with TS.

\section{Resting-state fMRI}

Resting-state fMRI is a method of functional brain imaging that can be used to evaluate regional interactions that occur when a subject is not performing an explicit task.

One study investigated alterations in spontaneous neural activity in children with TS and found suggestions of abnormal spontaneous neuronal activity and perturbed connectivity of cortico-subcortical networks in children with TS who had decreased spontaneous amplitude of low-frequency fluctuations (ALFF) and fractional ALFF in the posterior cingulated gyrus, precuneus and bilateral parietal gyrus, anterior cingulated cortex, bilateral middle and superior frontal cortices, and superior parietal lobule. ${ }^{38}$ Increased activity was seen in the left putamen and bilateral thalamus. ${ }^{38}$

\section{MEG}

MEG is a functional neuroimaging technique for mapping brain activity by recording magnetic fields produced by electrical currents occurring naturally in the brain by using very sensitive magnetometers. The MEG signals derive from the net effect of ionic currents flowing in the dendrites of neurons during synaptic transmission. 
Some studies have shown sensorimotor processing involvement in tic generation and in voluntary movement control. ${ }^{11,39}$ Sensorimotor processing refers to a process by which sensory information or input is coupled or integrated to a related motor response in the central nervous system. This process underlies both involuntary or reflexive actions and voluntary acts. One study used a combination of MEG and MRS (to be discussed in detail later) and found changes in oscillations in the sensory motor cortex in patients with TS compared with healthy controls, which might underpin some of the sensorimotor processing disturbances in TS and contribute to tic generation. ${ }^{11}$ Altered sensorimotor processing involved in voluntary movement control was also found in another study in which subjects with TS and healthy controls had to perform a go/no-go task and a movement task. ${ }^{39}$

In addition to the involvement of sensorimotor processing, another MEG study found increased functional interaction between SMA and M1 (primary motor cortex) during a task of voluntary movement control. ${ }^{40}$ The authors suggested that this might reflect a pathophysiological marker of TS. Another study investigating voluntary movements by a finger movement task found increased motor cortical activation in the contralateral hemisphere before and during movements and increased motor cortical inhibition in ipsilateral M1 after movements in patients with TS compared with healthy controls. ${ }^{41}$ Motor cortical reactivity might therefore be pathologically altered during voluntary movements in patients with TS. ${ }^{41}$

In summary, MEG studies have shown involvement of sensorimotor processing, motor cortical reactivity, and increased functional interaction between SMA and M1 in patients with TS.

\section{Functional connectivity}

FC refers to similar patterns of activation and relationship between spatially separated brain regions.

One study found that in children with TS, FC was abnormal in the bilateral prefronto-striatum-midbrain networks and in the bilateral sensorimotor and temporal cortices. ${ }^{42}$ The authors suggest that interhemispheric communication deficits might play a role in the pathophysiology of TS.

Werner et $\mathrm{al}^{43}$ found a significant increase in coupling between/within amygdalae in the TS group when compared to controls while performing a simple motor task. They suggest that the FC of amygdala must be altered in TS patients.

Some studies have suggested widespread immature FC and abnormal signaling, primarily in the frontoparietal network, both in a resting-state FC MRI study ${ }^{44}$ and during performance requiring adaptive control and task maintenance. ${ }^{45}$

Worbe et $\mathrm{al}^{46}$ found a stronger functional integration and a global functional disorganization of corticobasal ganglia networks in patients with TS compared with controls. They suggest that the pattern of the functional changes in corticobasal ganglia networks in patients could reflect a defect in brain maturation and that distinct regions of corticobasal ganglia networks could contribute to the clinical heterogeneity of TS.

In summary, changes in FC were seen in corticobasal ganglia networks, frontoparietal networks, prefronto-striatum-midbrain networks, sensorimotor and temporal lobes, and amygdala.

\section{TMS}

TMS is a magnetic method used to stimulate small regions in the brain. Several studies have described reductions of tic severity by repetitive TMS over the SMA, ${ }^{47-49}$ although there is no consensus. ${ }^{50}$

Bäumer et $\mathrm{al}^{51}$ found abnormal functional interhemispheric connectivity in TS accompanied by an altered structure-function relationship in the motor corpus callosum in a combined TMS and diffusion-tensor MRI study with stimuli in hand muscles. Pépés et a ${ }^{52}$ used TMS to examine cortical motor excitability in patients with TS and found that resting motor threshold and the variability of motor-evoked potential responses were significantly increased in children with TS, but these differences normalized with age. The authors suggest that these effects might be likely due to a developmental delay in the maturation of key brain networks in TS.

\section{MRS}

MRS is a noninvasive, ionizing-radiation-free analytical technique that has been used to study metabolic changes in the brain. This technique uses signals from hydrogen protons to determine the relative concentrations of target brain metabolites.

Two studies have examined the GABA concentrations in the sensorimotor cortex. ${ }^{11,53}$ The GABA-ergic system is the main inhibitory system in the CNS, and GABA-ergic neurons are present in every brain structure. ${ }^{54}$ Puts et $a l^{53}$ found reduced primary sensorimotor cortex GABA concentrations in children with TS compared to control subjects. These findings suggest that reduced GABA concentrations in TS may contribute to both motor tics and sensory impairments in children with TS. 
Tinaz et $\mathrm{al}^{11}$ did not find any differences in other GABA values in the right sensorimotor cortex between subjects with TS and healthy controls.

Another study found increased GABA concentrations within the SMA in individuals with TS compared to control subjects. $^{55}$

\section{NIRS}

NIRS is a spectroscopic method that uses the near-infrared region of the electromagnetic spectrum. The transmission and absorption of near-infrared light in human body tissues can provide information about hemoglobin concentration changes. One study suggests that children with TS might have reduced prefrontal hemodynamic response as measured by NIRS as a reduced hemoglobin concentration change was found in the prefrontal cortex of children with TS compared to healthy controls. ${ }^{56}$

\section{PET}

PET scans use a radioactive substance to observe metabolic processes in the body. Several PET studies have shown significant correlations between presence of tics and hypermetabolism in various brain regions, including medium and lateral premotor cortices, primary motor cortices, inferior parietal cortices, as well as anterior cingulate cortex, putamen, and caudate and Broca's area. ${ }^{57}$ These results support hyperactivity of the systems involved in motor planning/ structuring and in the processing of sensory inputs at the basis of TS symptoms. ${ }^{57}$ These findings have been partly confirmed by Pourfar et $\mathrm{a}^{58}$ who found a TS-related metabolic covariance pattern with increased activity bilaterally in premotor cortex and cerebellum, which was associated with relative reductions in striatum and orbitofrontal cortices. The activation of the sensorimotor circuits has also been shown in the study of de Vries et al, ${ }^{59}$ who propose that in TS patients, activation of the limbic structures enhances activation of sensorimotor circuits, which results in the emergence of an urge to perform tics. They found an increased regional blood flow in the inferior frontal gyrus, left anterior cingulate cortex, and SMA during provocation of symmetry behavior in patients with TS compared to healthy controls. Lerner et $\mathrm{al}^{60}$ has used a PET study in order to identify brain regions responsible for tic generation. Prominent activation during tic release was found in cerebellum and insula, and, furthermore, activation was seen in putamen, thalamus, SMA, and motor cortex. The authors suggest that the primary abnormality of TS originates with overactivity in the cerebellum, which influences the thalamus, basal ganglia, and multiple cortical areas, leading to tic production and premonitory sensation of urge. ${ }^{60}$

Dopaminergic, GABA, and serotonergic receptor systems have been thought to play a role in the pathophysiology of TS. One study examined the binding of GABA receptors and found decreased binding in the striatum, globus pallidus, thalamus, right insula, and amygdala and increased binding in substantia nigra, left periaqueductal gray, right posterior cingulate cortex, and bilateral cerebellar dentate nuclei. The authors suggest that the basal ganglia and thalamus are disinhibited in TS. ${ }^{54}$ With regard to the dopaminergic system, studies have shown contradictory results. Decreased dopamine receptor binding was found in patients with TS (both striatal ${ }^{61}$ and extrastriatal ${ }^{62}$ ). Steeves et $\mathrm{al}^{62}$ has found reductions in dopamine receptor binding in the frontal cortex and thalamus. Gilbert et $\mathrm{al}^{63}$ found reduced dopamine receptor availability in orbitofrontal and motor cortex, hippocampus, anterior cingulate gyrus, and mediodorsal thalamus. Some studies found indications for increased dopamine release in patients with TS compared with healthy controls. ${ }^{62,64}$ A couple of studies did not support a role for changes in striatal dopamine receptor availability or affinity in the pathophysiology of TS. ${ }^{65,66}$ Concerning serotonergic neurotransmission, one study found indications for increased serotonin synthesis in the caudate nucleus. ${ }^{67}$ The authors suggested that the abnormal connectivity between cortex and caudate nucleus may be the cause of abnormal neurotransmission in the corticostriato-thalamo-cortical circuit. ${ }^{67}$ Another study found a decreased tryptophan uptake in bilateral dorsolateral prefrontal cortex and increased tryptophan uptake in thalamus and subcortical structures to dorsolateral prefrontal cortex in patients with TS compared to healthy controls. ${ }^{68}$ Tryptophan is a precursor to the neurotransmitters serotonin, and thus this study suggested that impairment of cortical-subcortical serotonergic function plays a role in the pathophysiology of TS. ${ }^{68}$ Wong et al ${ }^{64}$ found serotonin transporter reductions in patients with TS with or without comorbid OCD, compared with healthy controls. Haugbol et al $^{69}$ found increased serotonin receptor binding in most brain regions.

Several studies suggested that an interaction between serotonin and dopamine might play a role in the pathophysiology of TS. ${ }^{64,69}$

In summary, PET studies have shown significant correlations between presence of tics and hypermetabolism in various brain regions. There are suggestions for abnormalities 
in the dopaminergic and serotonergic neurotransmission. Decreased binding of GABA receptors was found.

\section{SPECT}

SPECT is a nuclear medicine tomographic imaging technique. A marker radioisotope is attached to a specific ligand to create radio-ligands, which have the property of binding to certain types of tissues.

The dopamine transporter (DAT) is responsible for clearance of dopamine from the synaptic cleft after dopamine release. There are several DAT radio-ligands used in SPECT such as ${ }^{99 m}$ Tc-TRODAT-1, ${ }^{123} \mathrm{I}-\beta$-CIT, and ${ }^{123}$ I-FP-CIT.

However, findings based on imaging of DAT activity in patients with TS remain inconclusive.

One study showed significantly higher uptake of ${ }^{99 \mathrm{~m}} \mathrm{Tc}-$ TRODAT in drug-naive patients with TS compared with a control group. ${ }^{70}$ DAT uptake ratios were significantly negatively correlated with disease duration. The authors suggested that with the extension of the disease duration, a certain degree of adaptation might occur in the patients to compensate for DAT increase. ${ }^{70}$

This result was in contrast to a study by Yeh et al, ${ }^{71}$ who did not find significant difference in DAT activity between patients with TS and control subjects in the striatum and its subregions. They speculated whether this functional abnormality of the dopamine system in TS only exists in the early stage of the disease, although the mean age and mean duration of tics in the included patients was comparable in these two studies; in Liu et al's ${ }^{70}$ study the mean age was 24 years with a mean duration of tics of 13 years, while in Yeh et al's ${ }^{71}$ study, the mean age was 21.5 years and the mean duration of tics was 12.8 years.

Another study showed no significant difference in DAT and D2 receptor binding sites between TS patients and healthy controls. ${ }^{72}$

Yeh et $\mathrm{al}^{73}$ examined the effect of methylphenidate (MPH) pretreatment on DAT scanning. MPH is a central nervous stimulant that is used in the treatment of ADHD. DAT binding decreased significantly after MPH treatment both in drug-naive patients with TS and in healthy controls. The expected decline in DAT binding was, however, reduced in the TS group but only on the right striatum, indicating an overactive DAT system. Such a distinction was not found in the other striatal subregions in the two groups. The results suggested that the weakened blockade of DAT binding might reflect a functional abnormality of the dopaminergic system in the right caudate nucleus of TS patients.
In summary, SPECT studies suggested a functional abnormality of the dopamine system in patients with TS in its early stage. Adaptation to tic symptoms might play a role in regulating the neural system.

\section{EEG}

EEG is a noninvasive method to record electrical activity of the brain using electrodes placed along the scalp.

One study showed that during spontaneous tic expression, increased cortical oscillations of broad frequency bands in frontomotor areas were seen in children with TS compared with healthy controls. ${ }^{74}$ The neural oscillation was shown to propagate from the left prefrontal and centroparietal regions to the left frontal regions, which is suggested to be associated with the generation of tics. The voluntary expression of tics was associated with oscillations in the prefrontal region. The authors suggest that aberrant cortical oscillations might play a role in the pathophysiology of TS and that the frontal to motor interaction of cortical oscillations might play a role in tic suppression. Overactivation of cortical areas has also been suggested in a couple of other studies. ${ }^{75,76}$ It is hypothesized that this overactivation could be involved in both the control of tics and in the regulation of performance in adults with $\mathrm{TS}^{71}$ as well as in faster retrieval of required motor programs. ${ }^{76}$

Various EEG studies have examined the behavioral performance on several tasks. One study showed that children with TS might employ additional attention resources as a compensatory mechanism to maintain equal behavioral performance as healthy controls when tested with an Eriksen-Flanker test during EEG recording. ${ }^{77}$ The Eriksen-Flanker test is a set of response inhibition tests used to assess the ability to suppress responses that are inappropriate in a particular context. On the contrary, Thibault et $\mathrm{al}^{76}$ suggested that allocation of attention resources might delay stimulus evaluation and categorization processes. Yordanova et $\mathrm{al}^{78}$ found alterations in the processes of early selection in patients with TS and comorbid ADHD while performing an auditory selective attention task. These changes were not seen in patients with TS without ADHD, nor in patients with ADHD without TS or in healthy controls. Shephard et $\mathrm{al}^{79}$ found that TS was associated with slower reaction times in children with TS compared with healthy controls and children with ADHD while performing a go/no-go task during EEG recording. The authors suggest that reaction time slowing might represent a compensatory slowing of motor output to facilitate tics control.

In summary, EEG studies have shown aberrant cortical oscillations and indicate that patients with TS might employ 
extra attention resources, which could have an influence on their behavioral performance, and so they might have slower reaction times in order to compensate for their tics.

\section{Neuroimaging and severity of tics}

Several studies have examined the correlation between results of functional neuroimaging studies and severity of tics.

A significant correlation between severity of tics and blood-oxygen-level-dependent responses in the premotor cortices was seen in an fMRI study during performance of an imaginary motor task. ${ }^{34}$ The FC between the bilateral anterior cingulate cortex was found to be negatively correlated with tic severity. ${ }^{42}$ Another fMRI study suggested involvement of the frontostriatal circuits in the regulation of severity of tics. ${ }^{25}$ This finding was confirmed by Worbe et al, ${ }^{46}$ who found that functional abnormalities in all corticobasal ganglia networks (premotor, sensorimotor, parietal, and cingulated cortices and medial thalamus) were correlated with tic severity. Furthermore, they found that tic complexity was correlated with functional abnormalities in sensorimotor and associated networks, namely insula and putamen. ${ }^{46}$ One study suggested that decreased regional volumes of cerebellar hemispheres were correlated with increased tic severity. ${ }^{33}$ In an MRS study, it was shown that the GABA concentration in SM1 correlated negatively with motor tic severity. ${ }^{53}$ Amphetamineinduced dopamin release in the ventral striatum was related to increased tic severity in one PET study, ${ }^{61}$ while another PET study did not find any correlations between the TS-related metabolic covariance pattern (with increased activity bilaterally in premotor cortex and cerebellum, associated with relative reductions in striatum and orbitofrontal cortices) and severity of tics. ${ }^{58}$ During voluntary movement control measured with MEG, larger amplitudes were inversely correlated with motor tic frequency and severity, ${ }^{39}$ while another MEG study found ipsilateral motor cortical inhibition to be inversely related with tic severity. ${ }^{41}$ Yet another EEG study showed that increased cortical oscillations in frontomotor areas were correlated positively with tic severity. ${ }^{74}$

In summary, various functional neuroimaging methods have shown that changes in the cortico-thalamo-striatocortical pathways are correlated with tic severity.

\section{Perspectives and future studies}

In this review, we have described findings of various modalities of recent functional neuroimaging studies in patients with TS. Most of the recent studies have used fMRI, and this is also the only modality that has examined premonitory urges and tic suppression. fMRI studies have shown involvement of the cortico-striato-thalamo-cortical circuit and sensorimotor cortex in the generation of tic. The insula and sensorimotor cortex seem to be a part of the urge-tic network. During tic suppression, increased activity in the inferior frontal gyrus was seen. The premotor cortex might be involved in inhibition of motor control, and there is evidence for sensorimotor gating deficits. During regulation of motor output, activation of the prefrontal cortex might be increased. There seems to be evidence for altered motor network activations during voluntary motor execution. MEG studies have shown increased functional interaction between SMA and M1, motor cortical reactivity, and involvement of sensorimotor processing. Changes in FC were seen in corticobasal ganglia networks, frontoparietal network, prefronto-striatummidbrain networks, in sensorimotor and temporal cortices, and in amygdala. A TMS study has shown increased cortical motor excitability. MRS studies have suggested abnormalities in GABA concentrations, but the results are contradictory. A NIRS study has shown reduced prefrontal hemodynamic response. PET studies have suggested decreased binding of GABA receptors, abnormalities in the dopaminergic and serotonergic neurotransmission, and correlation between the presence of tics and hypermetabolism in various brain regions. SPECT studies have suggested a functional abnormality of the dopamine system in patients with TS in its early stage. EEG studies have shown aberrant cortical oscillations.

It can be difficult to compare neuroimaging studies due to various reasons.

First of all, the various modalities used different methods to examine neural activity in the brain. Changes in blood flow are used in fMRI and NIRS studies. The spatial resolution using NIRS is lower than that of fMRI and PET. Changes in metabolic processes in the brain, and thereby focus on function of the brain instead of structure, from the basis of PET and MRS studies. Radiation is used in PET, while MRS and fMRI do not use radiation. Electrical activity in the brain can be measured by MEG or EEG. Thereby, the results from the various modalities differ with regard to information about structure, function, and details in neuroanatomical substrates.

Secondly, not only do the modalities used differ among the included studies but also the used methods within the same modality can be different in the various studies. With regard to the fMRI studies, for example, one study has used resting state networks, ${ }^{12}$ while another has used regional homogeneity. ${ }^{19}$ The fMRI tasks themselves can have had different paradigms, ${ }^{21,22}$ but also the same tasks can have been performed in different ways - eg, finger tapping with preferred or nonpreferred hand or imaginary finger 
movements. ${ }^{30-32,34}$ Although methodological differences between studies can be small, they can cause different results, making the studies difficult to compare.

Furthermore, the age of the included subjects differed among the included studies. Some studies included children, ${ }^{29,30,38}$ some included adults, ${ }^{22,23}$ and some included both children and adults. Some modalities have only been performed with adult subjects, for instance the included FC, MEG, and PET studies. Since the phenotype of TS changes with age, it is difficult to draw any conclusions on the pathophysiology of TS based on neuroimaging studies using only cross-sectional designs. In some children, tics will disappear with age, and so these subjects will not be included as adults in adult cross-sectional studies since they do not have tics anymore. The adults included in cross-sectional studies who still have symptoms might represent another phenotypic variant of TS with another pathophysiologic mechanism compared with patients with remission of symptoms. ${ }^{80}$ To our knowledge, there has been only one longitudinal neuroimaging study on TS. ${ }^{80}$ A longitudinal study gives the opportunity to investigate the changes over time in the same patients from childhood into adulthood, and it can help examine the development of tics and comorbidity and correlate these to the neuroimaging findings. More longitudinal studies are warranted in the future in order to further examine the pathophysiological mechanisms of TS and the neuroplasticity that might be involved in TS.

In addition, not all studies have taken the presence of comorbidity into account in their analyses. With regard to MEG studies, for example, one study excluded neurological and psychiatric disorders except ADHD and OCD, ${ }^{11}$ while another study excluded ADHD and OCD. ${ }^{39}$ Studies on ADHD and OCD have shown the presence of neuroanatomical changes in these patients. In patients with OCD, abnormalities of cortico-striatal circuits that involve the orbitofrontal cortex, anterior cingulate cortex, prefrontal cortices, temporo-parieto-occipital areas, thalamus, and the striatum have been suggested, ${ }^{81,82}$ as well as impaired striatal connectivity. ${ }^{83}$ In patients with ADHD, dysfunctions in the cortico-striatal-thalamo-cortical circuits, ${ }^{84}$ changes in cortical thickness and gyrification, ${ }^{85}$ changes in the serotonin transporter binding potential, ${ }^{86}$ changes in callosal thickness, ${ }^{87}$ and reduced gray matter volumes in various regions of the brain are seen. ${ }^{88,89}$ In addition, the severity of ADHD and OCD symptoms probably has neuroanatomical correlates. ${ }^{81,82}$ Therefore, it is important to include presence of comorbidity in neuroimaging studies so as to be able to differentiate between neural changes caused by the presence of TS or by the presence of a comorbid disorder.
Moreover, not all studies have corrected for the use of medication. Some studies have included medicated patients, ${ }^{23,29,52}$ others only patients without medication. ${ }^{30}$ Also, the way of dealing with the use of medication has been different between the studies. For example, one study instructed the patients to discontinue medication 2 weeks prior to the study, ${ }^{11}$ while patients in other studies had to be off medical treatment for at least 6 months. ${ }^{39,40}$ Several studies have examined the influence of the use of medication on brain circuits. In patients with ADHD, long-term stimulant medication might normalize gray matter alterations. ${ }^{88}$ Neuroanatomical changes in OCD patients were stronger in medicated patients compared to controls in another study. ${ }^{90}$ A pharmaco-fMRI study has shown consistent and reproducible changes on disease-relevant networks and has proven sufficiently sensitive to detect dose-dependent network changes. ${ }^{91}$ This means that the use of medication needs to be taken into account when interpreting neuroimaging findings.

Finally, as described earlier, various changes in the cortico-thalamo-striato-cortical pathways seem to be correlated with severity of tics. Therefore, tic severity should be included in all neuroanatomical analyses.

Future neuroimaging studies on TS should include patients who are very well defined with regard to tic severity, presence and severity of comorbidity, and medical treatment, and these studies should include uniform inclusion and exclusion criteria. Longitudinal studies are warranted to enable disentanglement of the pathophysiology of TS. Further investigation of premonitory urge and tic suppression, which has only been examined in fMRI studies in the recent years, is needed, as it is important to focus on their neural correlates not only in the understanding of the pathophysiology of TS but also in the understanding of the mechanisms behind newer nonpharmacological treatments of TS, like behavioral therapy.

\section{Conclusion}

In this article, we reviewed the most recent functional neuroimaging studies on TS. We have included fMRI, MEG, FC, TMS, MRS, NRIS, PET, SPECT, and EEG studies. The most frequently performed functional neuroimaging studies during the last years have been fMRI and PET studies.

The cortico-striato-thalamo-cortical circuit and sensorimotor cortex seem to be involved in the generation of tics. There are suggestions for abnormalities of GABAergic, dopaminergic, and serotonergic neurotransmission in subjects with TS. Correlations have been found between presence of tics and hypermetabolism in various brain regions. There are 
suggestions that the insula is a part of the urge-tic network. During tic suppression, increased activity in the inferior frontal gyrus is seen. Various methods have shown changes in the cortico-thalamo-striato-cortical pathways to be correlated with tic severity. The premotor cortex might be involved in inhibition of motor control in patients with TS, but the results are contradictory. Several studies have shown altered motor network activations, and alterations in activation of the prefrontal cortex are suggested. Several studies have suggested sensorimotor gating deficits in subjects with TS.

It can be difficult to compare functional neuroimaging studies due to differences in methods, differences in age of the included subjects, and differences in the extent to which the presence of comorbidity, medical treatment, and severity of tics are considered in the various studies. In future studies, inclusion of more well-defined subjects and further examination of premonitory urge and tic suppression is needed in order to increase the knowledge about the pathophysiology and treatment possibilities for TS.

\section{Disclosure}

The authors report no conflicts of interest in this work.

\section{References}

1. American Psychiatric Association. The Diagnostic and Statistical Manual of Mental Disorders. 5th ed.; DSM-5. Arlington, VA: American Psychiatric Association; 2013.

2. Robertson MM. A personal 35 year perspective on Gilles de la Tourette syndrome: prevalence, phenomenology, comorbidities, and coexistent psychopathologies. Lancet Psychiatry. 2015;2(1):68-87.

3. Woods DW, Piacentini J, Himle MB, Chang S. Premonitory Urge for Tics Scale (PUTS): initial psychometric results and examination of the premonitory urge phenomenon in youths with Tic disorders. $J$ Dev Behav Pediatr. 2005;26(6):397-403.

4. Crossley E, Cavanna AE. Sensory phenomena: clinical correlates and impact on quality of life in adult patients with Tourette syndrome. Psychiatry Res. 2013;209(3):705-710.

5. McGuire JF, Ricketts EJ, Piacentini J, Murphy TK, Storch EA, Lewin AB. Behavior therapy for tic disorders: an evidenced-based review and new directions for treatment research. Curr Dev Disord Rep. 2015;2(4): 309-317.

6. Mol Debes N. Co-morbid disorders in Tourette Syndrome. Behav Neurol. 2013;27(1):7-14.

7. Khalifa N, Knorring AL. Prevalence of tic disorders and Tourette syndrome in a Swedish school population. Dev Med Child Neurol. 2003;45(5):315-319.

8. Scharf JM, Miller LL, Gauvin CA, Alabiso J, Mathews CA, Ben-Shlomo Y. Population prevalence of Tourette syndrome: a systematic review and meta-analysis. Mov Disord. 2015;30(2):221-228.

9. Robertson MM. The prevalence and epidemiology of Gilles de la Tourette syndrome. J Psychosom Res. 2008;65(5):461-472.

10. Crosson B, Ford A, McGregor KM, et al. Functional imaging and related techniques: an introduction for rehabilitation researchers. J Rehabil Res Dev. 2010;47(2):7-34.

11. Tinaz S, Belluscio BA, Malone P, van der Veen JW, Hallett M, Horovitz SG. Role of the sensorimotor cortex in Tourette syndrome using multimodal imaging. Hum Brain Mapp. 2014;35(12):5834-5846.
12. Neuner I, Werner CJ, Arrubla J, et al. Imaging the where and when of tic generation and resting state networks in adult Tourette patients. Front Hum Neurosci. 2014;8:362.

13. Bohlhalter S, Goldfine A, Matteson S, et al. Neural correlates of tic generation in Tourette syndrome: an event-related functional MRI study. Brain. 2006;129(Pt 8):2029-2037.

14. Hampson M, Tokoglu F, King RA, Constable RT, Leckman JF. Brain areas coactivating with motor cortex during chronic motor tics and intentional movements. Biol Psychiatry. 2009;65(7):594-599.

15. Rajagopal S, Seri And S, Cavanna AE. Premonitory urges and sensorimotor processing in Tourette syndrome. Behav Neurol. 2013;27(1): 65-73.

16. Tinaz S, Malone P, Hallett M, Horovitz SG. Role of the right dorsal anterior insula in the urge to tic in Tourette syndrome. Mov Disord. 2015; 30(9):1190-1197.

17. Draper A, Jackson GM, Morgan PS, Jackson SR. Premonitory urges are associated with decreased grey matter thickness within the insula and sensorimotor cortex in young people with Tourette syndrome. J Neuropsychol. 2016;10(1):143-153.

18. Kawohl W, Brühl A, Krowatschek G, Ketteler D, Herwig U. Functional magnetic resonance imaging of tics and tic suppression in Gilles de la Tourette syndrome. World J Biol Psychiatry. 2009;10(4 Pt 2):567-570.

19. Ganos C, Kahl U, Brandt V, et al. The neural correlates of tic inhibition in Gilles de la Tourette syndrome. Neuropsychologia. 2014;65:297-301.

20. Mazzone L, Yu S, Blair C, et al. FMRI study of frontostriatal circuits during the inhibition of eye blinking in persons with Tourette syndrome. Am J Psychiatry. 2010;167(3):341-349.

21. Ganos C, Kühn S, Kahl U, et al. Action inhibition in Tourette syndrome. Mov Disord. 2014;29(12):1532-1538.

22. Thomalla G, Jonas M, Bäumer T, et al. Costs of control: decreased motor cortex engagement during a Go/NoGo task in Tourette's syndrome. Brain. 2014;137(Pt 1):122-136.

23. Zapparoli L, Porta M, Paulesu E. The anarchic brain in action: the contribution of task-based fMRI studies to the understanding of Gilles de la Tourette syndrome. Curr Opin Neurol. 2015;28:604-611.

24. Jackson SR, Parkinson A, Jung J, et al. Compensatory neural reorganization in Tourette syndrome. Curr Biol. 2011;21(7):580-585.

25. Raz A, Zhu H, Yu S, et al. Neural substrates of self-regulatory control in children and adults with Tourette syndrome. Can J Psychiatry. 2009;54(9):579-588.

26. Marsh R, Zhu H, Wang Z, Skudlarski P, Peterson BS. A developmental fMRI study of self-regulatory control in Tourette's syndrome. Am J Psychiatry. 2007;164(6):955-966.

27. Baym CL, Corbett BA, Wright SB, Bunge SA. Neural correlates of tic severity and cognitive control in children with Tourette syndrome. Brain. 2008;131(Pt 1):165-179.

28. Zebardast N, Crowley MJ, Bloch $\mathrm{MH}$, et al. Brain mechanisms for prepulse inhibition in adults with Tourette syndrome: initial findings. Psychiatry Res. 2013;214(1):33-41.

29. Buse J, Beste C, Herrmann E, Roessner V. Neural correlates of altered sensorimotor gating in boys with Tourette Syndrome: a combined EMG/ fMRI study. World J Biol Psychiatry. 2016;17(3)187-197.

30. Debes NM, Hansen A, Skov L, Larsson H. A functional magnetic resonance imaging study of a large clinical cohort of children with Tourette syndrome. J Child Neurol. 2011;26(5):560-569.

31. Roessner V, Wittfoth M, Schmidt-Samoa C, Rothenberger A, Dechent P, Baudewig J. Altered motor network recruitment during finger tapping in boys with Tourette syndrome. Hum Brain Mapp. 2012;33(3): 666-675.

32. Roessner V, Wittfoth M, August JM, Rothenberger A, Baudewig J, Dechent P. Finger tapping-related activation differences in treatmentnaïve pediatric Tourette syndrome: a comparison of the preferred and nonpreferred hand. J Child Psychol Psychiatry. 2013;54(3): 273-279.

33. Tobe RH, Bansal R, Xu D, et al. Cerebellar morphology in Tourette syndrome and obsessive-compulsive disorder. Ann Neurol. 2010;67(4): $479-487$. 
34. Zapparoli L, Porta M, Gandola M, et al. A functional magnetic resonance imaging investigation of motor control in Gilles de la Tourette syndrome during imagined and executed movements. Eur J Neurosci. 2016;43(4):494-508.

35. Margolis A, Donkervoort M, Kinsbourne M, Peterson BS. Interhemispheric connectivity and executive functioning in adults with Tourette syndrome. Neuropsychology. 2006;20(1):66-76.

36. Neuner I, Kellermann T, Stöcker T, et al. Amygdala hypersensitivity in response to emotional faces in Tourette's patients. World J Biol Psychiatry. 2010;11(7):858-872.

37. Plessen KJ, Lundervold A, Grüner R, et al. Functional brain asymmetry, attentional modulation, and interhemispheric transfer in boys with Tourette syndrome. Neuropsychologia. 2007;45(4):767-774.

38. Cui Y, Jin Z, Chen X, He Y, Liang X, Zheng Y. Abnormal baseline brain activity in drug-naïve patients with Tourette syndrome: a resting-state fMRI study. Front Hum Neurosci. 2014;7:913.

39. Biermann-Ruben K, Miller A, Franzkowiak S, et al. Increased sensory feedback in Tourette syndrome. Neuroimage. 2012;63(1),119-125.

40. Franzkowiak S, Pollok B, Biermann-Ruben K, et al. Motor-cortical interaction in Gilles de la Tourette syndrome. PLOS ONE. 2012;7(1): e27850.

41. Franzkowiak S, Pollok B, Biermann-Ruben K, et al. Altered pattern of motor cortical activation-inhibition during voluntary movements in Tourette syndrome. Mov Disord. 2010;25(12):1960-1966.

42. Liao W, Yu Y, Miao HH, Feng YX, Ji GJ, Feng JH. Inter-hemispheric intrinsic connectivity as a neuromarker for the diagnosis of boys with Tourette syndrome. Mol Neurobiol. In press 2016.

43. Werner CJ, Stöcker T, Kellermann T, et al. Altered amygdala functional connectivity in adult Tourette's syndrome. Eur Arch Psychiatry Clin Neurosci. 2010;260(Suppl 2):S95-S99.

44. Church JA, Fair DA, Dosenbach NU, et al. Control networks in paediatric Tourette syndrome show immature and anomalous patterns of functional connectivity. Brain. 2009;132(Pt 1):225-238.

45. Church JA, Wenger KK, Dosenbach NU, Miezin FM, Petersen SE, Schlaggar BL. Task control signals in pediatric Tourette syndrome show evidence of immature and anomalous functional activity. Front Hum Neurosci. 2009;3:38.

46. Worbe Y, Malherbe C, Hartmann A, et al. Functional immaturity of cortico-basal ganglia networks in Gilles de la Tourette syndrome. Brain. 2012;135(Pt 6):1937-1946.

47. Kwon HJ, Lim WS, Lim MH, et al. 1-Hz low frequency repetitive transcranial magnetic stimulation in children with Tourette's syndrome. Neurosci Lett. 2011;492(1):1-4.

48. Le K, Liu L, Sun M, Hu L, Xiao N. Transcranial magnetic stimulation at 1 Hertz improves clinical symptoms in children with Tourette syndrome for at least 6 months. J Clin Neurosci. 2013;20(2):257-262.

49. Mantovani A, Leckman JF, Grantz H, King RA, Sporn AL, Lisanby SH. Repetitive Transcranial magnetic stimulation of the supplementary motor area in the treatment of Tourette Syndrome: report of two cases. Clin Neurophysiol. 2007;118(10):2314-2315.

50. Landeros-Weisenberger A, Mantovani A, Motlagh MG, et al. Randomized sham controlled double-blind trial of repetitive transcranial magnetic stimulation for adults with severe Tourette Syndrome. Brain Stimul. 2015;8(3):574-581.

51. Bäumer T, Thomalla G, Kroeger J, et al. Interhemispheric motor networks are abnormal in patients with Gilles de la Tourette syndrome. Mov Disord. 2010;25(16):2828-2837.

52. Pépés SE, Draper A, Jackson GM, Jackson SR. Effects of age on motor excitability measures from children and adolescents with Tourette syndrome. Dev Cogn Neurosci. 2016;19:78-86.

53. Puts NA, Harris AD, Crocetti D, et al. Reduced GABAergic inhibition and abnormal sensory symptoms in children with Tourette syndrome. J Neurophysiol. 2015;114(2):808-817.

54. Lerner A, Bagic A, Simmons JM, et al. Widespread abnormality of the gamma aminobutyric acid-ergic system in Tourette syndrome. Brain. 2012;135;1926-1936
55. Draper A, Stephenson MC, Jackson GM, et al. Increased GABA contributes to enhanced control over motor excitability in Tourette syndrome. Curr Biol. 2014;24(19):2343-2347.

56. Yamamuro K, Ota T, Iida J, et al. Prefrontal dysfunction in pediatric Tourette's disorder as measured by near-infrared spectroscopy. $B M C$ Psychiatry. 2015;15:102.

57. Alongi P, Iaccarino L, Perani D. PET neuroimaging: insights on dystonia and Tourette syndrome and potential applications. Front Neurol. 2014;5:183.

58. Pourfar M, Feigin A, Tang CC, et al. Abnormal metabolic brain networks in Tourette syndrome. Neurology. 2011;76:944-952.

59. De Vries FE, van den Heuvel OA, Cath DC, et al. Limbic and motor circuits involved in symmetry behavior in Tourette's syndrome. CNS Spectr. 2013;18(1):34-42.

60. Lerner A, Bagic A, Boudreau EA, et al. Neuroimaging of neuronal circuits involved in tic generation in patients with Tourette syndrome. Neurology. 2007;68(23):1979-1987.

61. Denys D, de Vries F, Cath D, Figee M, Vulink N, Veltman DJ. Dopaminergic activity in Tourette syndrome and obsessive-compulsive disorder. Eur Neuropsychopharmacol. 2013;23(11):1423-1431.

62. Steeves TD, Ko JH, Kideckel DM, et al. Extrastriatal dopaminergic dysfunction in tourette syndrome. Ann Neurol. 2010;67(2):170-181.

63. Gilbert DL, Christian BT, Gelfand MJ, Shi B, Mantil J, Sallee FR. Altered mesolimbocortical and thalamic dopamine in Tourette syndrome. Neurology. 2006;67(9):1695-1697.

64. Wong DF, Brasić JR, Singer HS, et al. Mechanisms of dopaminergic and serotonergic neurotransmission in Tourette syndrome: clues from an in vivo neurochemistry study with PET. Neuropsychopharmacology. 2008;33(6):1239-1251.

65. Abi-Jaoude E, Segura B, Obeso I, et al. Similar striatal D2/D3 dopamine receptor availability in adults with Tourette syndrome compared with healthy controls: A [(11) C]-(+)-PHNO and [(11) C]raclopride positron emission tomography imaging study. Hum Brain Mapp. 2015;36(7):2592-2601.

66. Albin RL, Koeppe RA, Wernette K, et al. Striatal [11C]dihydrotetrabenazine and $[11 \mathrm{C}]$ methylphenidate binding in Tourette syndrome. Neurology. 2009;72(16):1390-1396.

67. Saporta AS, Chugani HT, Juhász C, et al. Multimodality neuroimaging in Tourette syndrome: alpha-[11C] methyl-L-tryptophan positron emission tomography and diffusion tensor imaging studies. J Child Neurol. 2010;25(3):336-342.

68. Behen M, Chugani HT, Juhász C, et al. Abnormal brain tryptophan metabolism and clinical correlates in Tourette syndrome. Mov Disord. 2007;22(15):2256-2262.

69. Haugbøl S, Pinborg LH, Regeur L, et al. Cerebral 5-HT2A receptor binding is increased in patients with Tourette's syndrome. Int J Neuropsychopharmacol. 2007;10(2):245-252.

70. Liu H, Meng Z, Zhang B, Tan J, Wang Y. Evaluation of Tourette's syndrome by $99 \mathrm{mTc}$-TRODAT-1 SPECT/CT imaging. Ann Nucl Med. 2010;24(7):515-521.

71. Yeh CB, Lee CH, Chou YH, Chang CJ, Ma KH, Huang WS. Evaluating dopamine transporter activity with 99mTc-TRODAT-1 SPECT in drug naïve Tourette's adults. Nucl Med Commun. 2006;27:779-784.

72. Hwang WJ, Yao WJ, Fu YK, Yang AS. 99mTc-TRODAT-1/[123 I]IBZM SPECT studies of the dopaminergic system in Tourette Syndrome. Psychiatry Res. 2008;162(2):159-166.

73. Yeh CB, Lee CS, Ma KH, et al. Phasic dysfunction of dopamine transmission in Tourette's syndrome evaluated with 99m TC TRODT-1 imaging. Psychiatry Res. 2007;156(1):75-82.

74. Hong HJ, Sohn H, Cha M, et al. Increased frontomotor oscillations during tic suppression in children with Tourette syndrome. $J$ Child Neurol. 2013;28(5):615-624.

75. Thibault G, Felezeu M, O'Connor KP, Todorov C, Stip E, Lavoie ME. Influence of comorbid obsessive-compulsive symptoms on brain event-related potentials in Gilles de la Tourette syndrome. Prog Neuropsychopharmacol Biol Psychiatry. 2008;32(3):803-815. 
76. Thibault G, O’Connor KP, Stip E, Lavoie ME. Electrophysiological manifestations of stimulus evaluation, response inhibition and motor processing in Tourette syndrome patients. Psychiatry Res. 2009; 167(3):202-220.

77. Eichele H, Eichele T, Bjelland I, et al. Performance monitoring in medication-naïve children with Tourette Syndrome. Front Neurosci. 2016;10:50.

78. Yordanova J, Heinrich H, Kolev V, Rothenberger A. Increased eventrelated theta activity as a psychophysiological marker of comorbidity in children with tics and attention-deficit/hyperactivity disorders. Neuroimage. 2006;32(2):940-955.

79. Shephard E, Jackson GM, Groom MJ. The effects of co-occurring ADHD symptoms on electrophysiological correlates of cognitive control in young people with Tourette syndrome. J Neuropsychol. In press 2015.

80. Debes NM, Jeppesen SS, Raghava J, et al. Longitudinal MRI analysis of the developmental changes of Tourette Syndrome reveal reduced diffusion in the cortico-striato-thalamo-cortical pathways. J Child Neurol. 2015;30(10):1315-1326.

81. Glahn A, Prell T, Grosskreutz J, Peschel T, Müller-Vahl KR. Obsessivecompulsive disorder is a heterogeneous disorder: evidence from diffusion tensor imaging and magnetization transfer imaging. $B M C$ Psychiatry. 2015;15:135.

82. Piras F, Piras F, Chiapponi C, Girardi P, Caltagirone C, Spalletta G. Widespread structural brain changes in OCD: a systematic review of voxel-based morphometry studies. Cortex. 2015;62:89-108.

83. Bernstein GA, Mueller BA, Schreiner MW, et al. Abnormal striatal resting-state functional connectivity in adolescents with obsessivecompulsive disorder. Psychiatry Res. 2016;247:49-56.
84. Zhu Y, Yang D, Ji W, et al. The Relationship between neurocircuitry dysfunctions and attention deficit hyperactivity disorder: a review. Biomed Res Int. In press 2016.

85. Mous SE, White T, Muetzel RL, et al. Cortical morphology as a shared neurobiological substrate of attention-deficit/hyperactivitysymptoms and executive functioning: a population-based pediatric neuroimaging study. J Psychiatry Neurosci. In press 2016.

86. Vanicek T, Kutzelnigg A, Philippe C, et al. Altered interregional molecular associations of the serotonin transporter in attentiondeficit/ hyperactivity disorder assessed with PET. Hum Brain Mapp. In press 2016.

87. Luders E, Kurth F, Das D, et al. Associations between corpus callosum size and ADHD symptoms in older adults: The PATH through life study. Psychiatry Res. 2016;256:8-14.

88. Moreno-Alcázar A, Ramos-Quiroga JA, Radua J, et al. Brain abnormalities in adults with attention deficit hyperactivity disorder revealed by voxel-based morphometry. Psychiatry Res. 2016;254:41-47.

89. He N, Li F, Li Y, et al. Neuroanatomical deficits correlate with executive dysfunction in boys with attention deficithyperactivity disorder. Neurosci Lett. 2015;600:45-49.

90. Boedhoe PS, Schmaal L, Abe Y, et al. Distinct subcortical volume alterations in pediatric and adult OCD: a worldwide meta- and megaanalysis. Am J Psychiatry. In press 2016.

91. Wandschneider B, Koepp MJ. Pharmaco fMRI: determining the functional anatomy of the effects of medication. Neuroimage Clin 2016;12:691-697.

92. Singer HS, Mink JW, Gilbert DL, Jankovic J. Movement Disorders in Childhood. 2nd ed. Philadelphia, PA: Elsevier Inc; 2016.
Neuroscience and Neuroeconomics

\section{Publish your work in this journal}

Neuroscience and Neuroeconomics is an international, peer-reviewed, open access journal focusing on the identification of brain structures and measurement of neural activity related to behavior, behavioral predictions, and decision making in health and disease. The manuscript

\section{Dovepress}

management system is completely online and includes a very quick and fair peer-review system. Visit http://www.dovepress.com/testimonials. php to read real quotes from published authors. 